'Privacy and Confidentiality'. The learning objectives were met for the majority of participants. Different aspects of the modules - format, references, learning activities were rated from 1 (worst) to 5 (best) with all ratings ranging from 3 to 5 . Areas for improvement were video time length as some rural areas had difficulty with internet speed but the majority were perceived to be relevant to rural practice and the mode of learning acceptable for practitioners.

Conclusion The modules promote inquiry-based learning for adult learners and provide opportunity for rural doctors to critique and reflect on their own practice.

Disclosure No significant relationships.

\section{P329 STRESS, POST-TRAUMATIC STRESS DISORDER, AND SEXUAL AND REPRODUCTIVE HEALTH IN A NATIONALLY-REPRESENTATIVE SAMPLE OF US WOMEN}

Joy Scheidel"*, Maria Khan. NYU School of Medicine, New York, USA

10.1136/sextrans-2019-sti.439

Background Examination of stress and post-traumatic stress disorder (PTSD) in relation to sexual and reproductive health $(\mathrm{SRH})$ is limited. Stress, potentially by impairing immune response and increasing risk-taking behavior, may influence risk of poor SRH. 'Weathering' effects from chronic stress exposure that accumulate over time may further heighten risk.

Methods Using Wave IV data on 7870 women (ages 24-34) in the National Longitudinal Study of Adolescent to Adult Health, we defined high stress as scores $\geq 6$ ( $75^{\text {th }}$ percentile) on the 4-item Perceived Stress Scale and PTSD as self-reported diagnosis by a healthcare provider; we categorized years since diagnosis as never, $\leq 2,3-5, \geq 6$. Women reported lifetime and past year sexually transmitted infection (STI) and pelvic inflammatory disease (PID) diagnoses, adverse pregnancy outcomes (miscarriage, ectopic/tubal pregnancy, stillbirth), and impaired fecundity (difficultly becoming/staying pregnant). We used modified Poisson regression to estimate sociodemographic-adjusted prevalence ratios (APR) and 95\% confidence intervals (CI).

Results Approximately 43\% reported high stress and 4\% PTSD ( $1 \% \leq 2$ years since diagnosis, $1 \% 3-5$ years, $2 \% \geq 6$ years). High stress was associated with past year bacterial STIs $(A P R=1.53,95 \% \mathrm{CI}: 1.13,2.09)$, unassociated with viral STIs, and weakly associated with adverse pregnancy outcomes $(\mathrm{APR}=1.24,95 \% \mathrm{CI}: 1.02,1.50)$ and impaired fecundity $(\mathrm{APR}=1.13$, 95\%CI: 0.98, 1.30). PTSD was also associated with lifetime viral STIs $(\mathrm{APR}=1.40,95 \% \mathrm{CI}$ : 1.06, 1.83) and PID (APR $=3.12,95 \% \mathrm{CI}: 1.59,6.12)$. Compared to women never diagnosed with PTSD, APRs for past year bacterial STIs were 2.66 (95\%CI: 1.28, 5.55) and 3.12 (95\%CI: $1.49,6.55)$ for women diagnosed $\leq 2$ years and $\geq 6$ years ago respectively, but not associated for women diagnosed 3-5 years ago.

Conclusion High 'global' perceived stress is associated with increased prevalence of poor SRH among women. Stronger associations were demonstrated in women with PTSD, particularly those with shorter and longer time since diagnosis, potentially suggesting both adverse acute and chronic stress responses.

Disclosure No significant relationships.

\section{P330 \\ THE SEXUAL AND REPRODUCTIVE HEALTH NEEDS OF HARD TO REACH GROUPS IN UGANDA}

Samuel Mukasa*, Peter Byansi. Uganda Martrys University, Health Sciences, Kampala, Uganda

\subsection{6/sextrans-2019-sti.440}

Background Inorder to achieve universal health coverage for all, the government of Uganda committed to advancing family planning for all during the FP2020 London Summit in July 2017. Despite efforts by Ministry of health (MOH) and Implementing partners (IPs) to implement programs that are complementary to static services, some communities remain hard to reach. Emerging New groups of nomads, balaro (Agago, Gulu, Apach). These don't return but settle in new scarcely populated areas, father children, acquire STI The Ike suffer from attacks from Kenya and Uganda, are isolated, not reached by SRHR services, have SRH needs. The Batwa, lived in forests but now displaced. Lost livelihood, Not integrating well with communities. Sexually exploited by communities due to myths. These groups remain hard to reach by due to factors like; stigma, geographical isolation, cultural beliefs, life style patterns and biological factors. There's been minimal change in SRH indicators, despite growing focus by IP (Kanungu, Kisoro, Rubanda)

Methods A qualitative design utilizing case study approach to qualitative inquiry was used Data collection was by; Document review Key informant interviews (KIIs) Focus group discussions (FGDs) Sampling for FGDs and KIIs was purposive with maximum variation Data collected was transcribed, analysed thematically and report generated

Results The key SRHR needs include; sexually transmitted infections, sexual and gender-based violence, family planning, female genital mutilation, limited delivery at health facilities, low ANC attendance and a high held role of TBAs in these communities. The most significant barriers to SRHR services include; extreme poverty, poor cultural beliefs, low literacy levels, alcohol abuse, language barriers, distance between the clients and available heath services.

Conclusion The SRHR needs are documented in the existing literature and not unique to hard to reach groups though of greter impacts among these groups. The most unique barrier to SRH services incude; language barrier, poverty and distance to existing health services

Disclosure No significant relationships.

\section{P331 QUANTITATIVE EVALUATION OF AN INNOVATION CONTEST TO ENHANCE A SEXUAL HEALTH CAMPAIGN IN CHINA}

${ }^{1}$ Ye Zhang ${ }^{*},{ }^{2}$ Songyuan Tang, ${ }^{3}$ Katherine Li, ${ }^{4}$ Lai Sze Tso, ${ }^{5}$ Barry Bayus, ${ }^{6}$ David Glidden, ${ }^{7}$ Bin Yang, ${ }^{8}$ He-Ping Zheng, ${ }^{9}$ Chongyi Wei, ${ }^{4}$ Joseph Tucker, ${ }^{4}$ Weiming Tang. ${ }^{1}$ Kirby Institution, UNSW, Sydney, Australia; ${ }^{2}$ Kunming Medical University, Kunming, China; ${ }^{3}$ Weill Cornell Medical College, New York, USA; ${ }^{4}$ UNC Project-China, Guangzhou, China; ${ }^{5}$ University of North Carolina, Chapel Hill, USA; ${ }^{6}$ University of California, San Francisco, USA; 'Guangdong Center for STD Control and Prevention, Guangzhou, China; ${ }^{8}$ Dermatology Hospital of Southern MedicalUniversity, Guangdong Center for STD Control and Prevention, Guangzhou, China; ${ }^{9}$ The State University of New Jersey, Rutgers, USA

\subsection{6/sextrans-2019-sti.441}

Background Crowdsourcing method is an excellent tool for developing tailored interventions to improve sexual health. We 
evaluated the implementation of an innovation contest for sexual health promotion in China.

Methods We organized an innovation contest over three months in 2014 for Chinese individuals $<30$ years old to submit images for a sexual health promotion campaign. We solicited entries via social media and in-person events. The winning entry was adapted into a poster and distributed to STD clinics across Guangdong Province. In this study, we evaluated factors associated with images that received higher scores, described the themes of the top five finalists, and evaluated the acceptability of the winning entry using an online survey tool.

Results We received 96 image submissions from 76 participants in 10 Chinese provinces. Most participants were youth $(<25$ years, 85\%) and non-professionals (without expertise in medicine, public health or media, $88 \%$ ). Youth were more likely to submit high-scoring entries. Images from professionals did not have higher scores compared to images from non-professionals. Participants were twice as likely to have learned about the contest through in-person events compared to social media. We adapted and distributed the winning entry to 121 public STI clinics over 2 weeks. A total of 8338 people responded to an acceptability survey of the finalist entry. The majority of the survey respondents found the winning image acceptable and engaging, with $43 \%$ of respondents strongly endorsing and $43.4 \%$ of respondents endorsing approval of the image. Additionally, $79.8 \%$ endorsed or strongly endorsed being more willing to undergo STD testing after seeing the poster.

Conclusion Innovation contests may useful for soliciting images as a part of comprehensive sexual health campaigns in low- and middle-income countries. Future sexual health campaigns should incorporate face-to-face interactions where participants can ask questions and solicit feedback about their submission ideas.

Disclosure No significant relationships.

\section{P334 ACCEPTANCE OF CONTRACEPTIVE VAGINAL RING AMONG WOMEN WITH BACTERIAL VAGINOSIS AND THEIR MALE PARTNERS IN KENYA}

${ }^{1}$ Kenneth Ngure, ${ }^{2}$ Kate Heller, ${ }^{3}$ ssekah Osang'Ir, ${ }^{1}$ Elizabeth Irungu, ${ }^{4}$ Katherine Thomas, ${ }^{2}$ Lei Wang, ${ }^{2}$ Meighan Krows, ${ }^{3}$ Nelly Mugo, ${ }^{5}$ Jeanne Marrazzo*. ${ }^{1}$ Jomo Kenyatta University of Agriculture and Technology, Community Health, Nairobi, Kenya; ${ }^{2}$ University of Washington, Global Health, Seattle, USA; ${ }^{3}$ Kenya Medical Research Institute, Center for Clinical Research, Nairobi, Kenya; ${ }^{4}$ University of Washington, Seattle, USA; ${ }^{5}$ University of Alabama, USA

\subsection{6/sextrans-2019-sti.442}

Background Multiple studies have shown that changes in the vaginal microbiome associated with increased risk of bacterial vaginosis (BV) are a risk for HIV acquisition and transmission. Therefore, hormonal interventions that prevent unintended pregnancies and promote vaginal health could reduce this risk. However, there is little data on acceptability of intravaginal rings in sub-Saharan Africa countries. We assessed the acceptance of a contraceptive vaginal ring (CVR) among women with BV and their male partners in Thika, Kenya.

Methods Between April 2016 to November 2017, we enrolled and treated women with BV aged 18-40 years. One month later, they were randomized to cyclical or continuous CVR and followed up monthly for 7 months. Additionally, we interviewed a sample of their male partners on their perspectives of the CVR. We conducted Fisher's exact tests for each category of baseline demographics by whether or not women accepted to be randomized to CVR use and used descriptive statistics to summarize the views of their male partners.

Results A total 149 women were enrolled and 122 (81.9\%) initiated CVR at the randomization visit. Reasons for not initiating CVR were, 17 (11.4\%) did not return for randomization and were considered lost to follow-up, 6 (4\%) expressly refused to use CVR, and $4(2.7 \%)$ terminated due to other reasons. There were no differences between randomized and non-randomized women except by pre-specified categories of number of children $(p=0.02)$. Of the 32 male partners that were interviewed, $96.9 \%$ were fine with their partners using the CVR, $78.1 \%$ reported that the ring was a very effective contraceptive method while $3.1 \%$ reported that it caused physical discomfort during sex.

Conclusion There was high acceptability of CVR among women with BV and their male partners in Kenya. Therefore, vaginal rings are a promising strategy that should be evaluated for delivery of multipurpose prevention in Kenyan women.

Disclosure No significant relationships.

\section{P335 E-DEVICES USAGE IN ORDER TO PROMOTE MHEALTH FOR YOUNG KEY POPULATION: HACKHEALTH OUTCOMES}

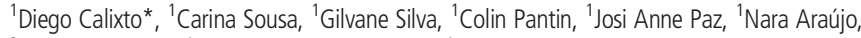
${ }^{2}$ Adele Benzaken, ${ }^{1}$ Gerson Fernando Pereira. ${ }^{1}$ Ministry of Health of Brazil, Department of Surveillance, Prevention and Control of STIs, HIVIAIDS and Viral Hepatitis, Brasilia, Brazil; ${ }^{2}$ Ministerio da Saúde do Brasil, Brazil

10.1136/sextrans-2019-sti.443

Background In Brazil, young people aged 15-24yo represent 27.4\% of all new HIV infections. Brazilian Ministry of Health of Brazil has launched the HackHealth Project to develop innovative e-devices capable of increasing knowledge about sexual health and combination prevention among young people. Hackhealth is an innovative, dynamic and open knowledge-based project. Our aim is to describe the results.

Methods Young key population (YKP) were recruited through a nationwide questionnaire and randomly assigned into 5 groups, being 5 YKP, 2 programmers and 1 graphic designer. The project lasted 36 hours and each group elected a leader.

Results 5 innovative e-devices were developed - a game about sex education, quiz with gifs, sexual health app, crowdsourcing platform, and a chatbot on combination prevention for the official website of the Ministry of Health of Brazil. The project focused on engaging young people in Information and Communication Technologies (ICT), while expanding their knowledge on combined prevention and sexual health.

Conclusion 5 innovative e-devices were developed - a game about sex education, quiz with gifs, sexual health app, crowdsourcing platform, and a chatbot on combination prevention for the official website of the Ministry of Health of Brazil. The project focused on engaging young people in Information and Communication Technologies (ICT), while expanding their knowledge on combined prevention and sexual health. Disclosure No significant relationships. 